\title{
Fluorescent Derivatization of Nitrite Ions with 2,3-Diaminonaphthalene Utilizing a pH Gradient in a Y-shaped Microchannel
}

\author{
Tamao OdaKe,* Mari TabuChI,* Takanori Sato,** Hironori SuSAKI,** \\ and Takashi KorenaGA $* * * * \dagger$ \\ *Department of Ecosystem Engineering, Graduate School of Engineering, University of Tokushima, \\ 2-1 Minami-josanjima, Tokushima 770-8506, Japan \\ **Elemental Technology Development and Researching Laboratory, Furuno Electric Co., Ltd., \\ 9-52 Ashihara, Nishinomiya 662-8580, Japan \\ ***Faculty of Integrated Arts and Sciences, University of Tokushima, \\ 1-1 Minami-josanjima, Tokushima 770-8502, Japan
}

\begin{abstract}
The on-chip derivatization of nitrite ions with 2,3-diaminonaphthalene (DAN) utilizing a $\mathrm{pH}$ gradient formed in a Yshaped microchannel was investigated. Nitrite ions react with $\mathrm{DAN}$ at low $\mathrm{pH}$, and strongly fluoresced at high $\mathrm{pH}$. Therefore, a reaction at low $\mathrm{pH}$ followed by the addition of a strong alkaline solution is the usual procedure in a batch scheme. However, a strong alkaline solution, like an $\mathrm{NaOH}$ aqueous solution, erodes the wall of the microchannels in substrates made of glass or polymers, and has not been considered suitable for use in microchannels. We first investigated the derivatization reaction and fluorescent properties of nitrite ions with DAN. We found that the on-chip fluorescent derivatization reaction and detection without the addition of an alkaline solution is possible by controlling the $\mathrm{pH}$ values of the nitrite solution and the DAN solution to form a suitable $\mathrm{pH}$ gradient by utilizing a buffering effect of triethanolamine solution, which is used as an $\mathrm{NO}_{2}$ gas-absorption medium. These results have suggested the feasibility of novel reaction schemes which can provide the desired products due to a controlled $\mathrm{pH}$ gradient in the microchannels, as well as the possibility of an on-site monitoring microchip device for ambient $\mathrm{NO}_{2}$.
\end{abstract}

(Received December 4, 2000; Accepted January 21, 2001)

\section{Introduction}

Recent advances in microchip analysis have been most remarkable since the conception of a micro total-analysis system $(\mu \text {-TAS })^{1}$ and a lab-on-a-chip, ${ }^{2}$ or chemistry integration being proposed. One of the well-known advantages of $\mu$-TAS is a downsizing of the experimental equipment, which leads to on-site monitoring as well as high-speed, high-throughput analysis, resulting from a small sample volume and a small experimental space. The initial applications of microchip analysis were to miniaturize capillary electrophoresis. Also, for example, amino acids, ${ }^{3-6}$ proteins, ${ }^{7-10}$ and DNA fragments ${ }^{11-14}$ were electrophoretically separated at high speed. For example, Effenhauser et al. demonstrated the on-chip $14 \mathrm{~s}$ separation of a mixture of six FITC-labeled amino acids. ${ }^{4}$ Integration of the analytical system is also one of the advantages of $\mu$-TAS, and DNA chips ${ }^{15,16}$ including PCR-based DNA analysis chips $^{17-20}$ have been developed.

Another advantage of $\mu$-TAS is the possibility of novel, highperformance analytical systems utilizing techniques only possible at microscales. In $150 \mu \mathrm{m}$ wide microchannels, for example, the mixing time was estimated to be about $20 \mathrm{~s}$, which

$\doteqdot$ To whom correspondence should be addressed.

T. O. present address: Department of Chemistry, Gunma University, Kiryu 376-8515, Japan. corresponds well to the molecular diffusion time. ${ }^{21}$ Therefore, provided that mixing is performed only by diffusion, the smaller is the width of the microchannel, the faster can be the reaction completed. By using the fast mixing and diffusion properties of microchip analysis, an analytical system can be integrated into a chip with high performance. For example, flow-injection analysis was integrated on a single microchip, and the Fe(II)-ophenanthroline complex was determined at zeptomole levels. ${ }^{21}$ The observed mixing time was about $20 \mathrm{~s}$, which corresponded well to the calculated value, with the diffusion length assumed to be the channel size. Furthermore, a solvent-extraction system was also integrated on a glass microchip, ${ }^{22}$ and the $\mathrm{Fe}$ bathophenanthrolinedisulfonic acid complex in aqueous solution was extracted in chloroform by forming an ion-pair product.

Thus, the $\mu$-TAS techniques have possibilities of providing novel analytical devices with high performance. The typical difference between the usual chemical analysis and microchip analysis may be the interface area-to-volume ratio. During both mixing and extraction, molecular transport occurs at the interface of two liquids, i.e., a liquid-liquid interface. The interface area-to-volume ratio was estimated to be $80 \mathrm{~cm}^{-1}$ in the case of microchannels $250 \mu \mathrm{m}$ wide and $100 \mu \mathrm{m}$ deep, while it was about $10^{0}-10^{1} \mathrm{~cm}^{-1}$ in the case of using an ordinary separatory funnel. ${ }^{22}$ Therefore, the specific interface area is 8 80 times larger in microchannels than in separatory funnels, and faster mixing by molecular transport is expected in 


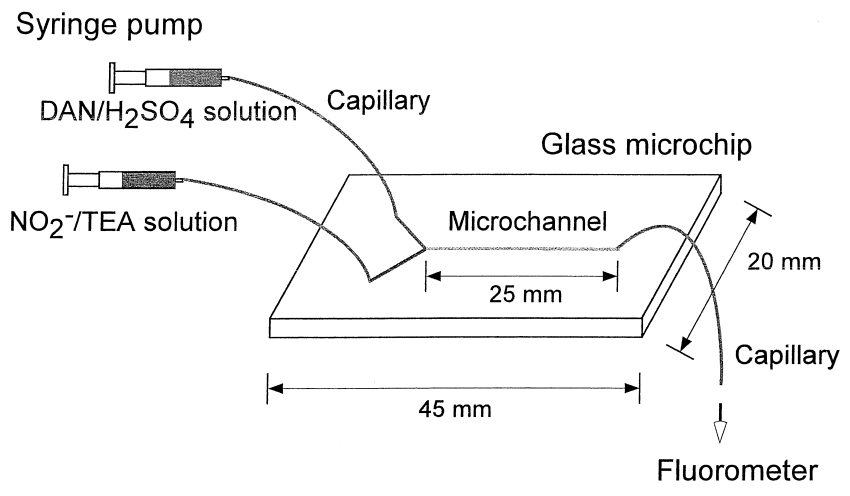

Fig. 1 Schematic illustration of the experimental setup for fluorescent derivatization in microchannel flow.

microchannels than in separatory funnels.

In microchips, the interface plays a more important role than in the usual chemical procedures. However, few researchers have paid attention to the nonuniformity that is generated in the vicinity of the interfacial region of two liquids, though the mixing time, determined by molecular diffusion, is very short. When two substances react, mixing is needed, and complete mixing as soon as possible is considered to be best. In a microchip reaction as well as FIA, fast mixing is considered to be preferable. However, in microchannels, where the specific interface area is inevitably large compared to the bulk, a nonuniform distribution of substances, which leads to $\mathrm{pH}$ gradients, has a feasibility to provide novel analytical procedures.

Nitrite is a nitrogen compound, the determination of which is essential in environmental chemistry, geochemistry and limnology. Nitrogen dioxide, which is produced by vehicles, and is one of the major sources of environmental pollution, can be solved in a gas-absorption solution, such as a triethanolamine (TEA) solution, and exists as nitrite. In the determination of nitrite in microchannels, sensitive detection should be performed. Because fluorometry is one of the most sensitive determination methods, fluorometric determination methods of nitrite have been developed and reviewed in the literature. ${ }^{23}$ Among many fluorescent reagents for nitrite, 2,3diaminonaphthalene (DAN) seems to be preferable, because the wavelength of fluorescence excitation is close to the emission wavelength of a UV light-emitting diode (LED), which has maximum emission intensity at $370 \mathrm{~nm}$. LEDs are suitable for a chip-based miniaturized system, and fluorescent detection has been expected to obtain sufficient sensitivity, even with the weak intensity of the LED, and in the small detection volume in microchannels. However, nitrite ions effectively react with DAN in an acidic medium, giving 2,3-naphthotriazole (NTA), which is highly fluorescent in an alkaline medium. Therefore, in the usual batch detection scheme, a strong alkaline solution, such as an $\mathrm{NaOH}$ aqueous solution, is added to the NTA solution to obtain high sensitivity. However, a strong alkaline solution will erode the wall of microchannels in a substrate, such as glass or polymers, and the addition of such an alkaline solution is undesirable for microchannels in a glass substrate. Therefore, a novel reaction scheme for the detection of nitrite without the need to add an alkaline solution has been desired.

We investigated an on-chip fluorescent derivatization reaction scheme utilizing a $\mathrm{pH}$ gradient formed in the vicinity of an interfacial region. First, the reaction with DAN and the fluorescent efficiencies of NTA for nitrite determination were investigated regarding a batch scheme. A simple-type Y-shaped microchannel was used to mix the two solutions: DAN dissolved in an acidic solution and nitrite in a triethanolamine (TEA) solution. By utilizing a buffering effect of TEA and the $\mathrm{pH}$ gradient formed in the microchannel, nitrite ions proved to be effectively derivatized with DAN, and were determined by successive fluorescence detection without the addition of a strong alkaline solution.

\section{Experimental}

\section{Microchip with Y-shaped microchannel}

A schematic illustration of the experimental setup for a microchannel reaction is shown in Fig.1. Microchips with a Yshaped microchannel were obtained from Technology Research Laboratory, Shimadzu Corp. (Kyoto, Japan). The substrate of the microchip was a $45 \mathrm{~mm} \times 20 \mathrm{~mm}$ quartz glass plate, and the thickness was $2 \mathrm{~mm}$. The Y-shaped microchannel was fabricated by photo lithography and a wet chemical-etching method. ${ }^{24}$ The microchannel was $c a .200 \mu \mathrm{m}$ wide and $20 \mu \mathrm{m}$ deep. A DAN solution and a nitrite solution were flowed into the microchannel using two syringe pumps with a flow rate of $0.04 \mu \mathrm{m} / \mathrm{min}$ each, which corresponded to a linear velocity of 2 $\mathrm{cm} / \mathrm{min}$ in the mixed region. The two solutions mixed in the microchannel and a drain solution was collected through a fused silica capillary, and fed into a fluorescence spectrophotometer (RF-5300, Shimadzu) with a flow cell having a $250 \mu \mathrm{m}$ long optical path (Ex. $375 \mathrm{~nm}$, Em. $425 \mathrm{~nm}$ ).

\section{Samples and reagents}

Nitrite samples were prepared by dissolving sodium nitrite in a $3 \%(w / v)$ TEA aqueous solution. DAN was solved in $0.25 \mathrm{M}$ sulfuric acid. In batch-scheme experiments, $2 \mathrm{ml}$ each of a nitrite solution and a DAN solution were mixed, and then incubated for $10 \mathrm{~min}$. Before a fluorescent measurement, appropriate amounts of a $1 \mathrm{M} \mathrm{NaOH}$ aqueous solution were added to the mixed solutions while adjusting the $\mathrm{pH}$.

DAN was purchased from Dojindo Laboratories (Kumamoto, Japan) and TEA was from Wako (Osaka, Japan). All reagents were of reagent grade and distilled water was used.

\section{Results and Discussion}

\section{Fluorescent derivatization property of nitrite ions}

The derivatization reaction and fluorescent properties of nitrite ions were first investigated using a batch scheme. The fluorescent derivatization of nitrite ions with DAN depends on the $\mathrm{pH}$ value. Nitrite ions effectively react with DAN in an acidic $(\mathrm{pH}<2)$ media, and generated NTA gives stronger fluorescence in alkaline $(\mathrm{pH}>10)$ media than in acidic media. The fluorescence intensity of nitrite ions derivatized with DAN at different reaction and detection $\mathrm{pH}$ is shown in Fig. 2. As predicted, a low reaction $\mathrm{pH}(\mathrm{pH} 2)$ and high detection $\mathrm{pH}(\mathrm{pH}$ 13) condition gave the highest sensitivity, and either a high reaction $\mathrm{pH}$ or a low detection $\mathrm{pH}$ condition gave insufficient sensitivity to detect nitrite ions in the range of $10^{-7} \mathrm{M}$. However, the condition of reaction $\mathrm{pH} 2$ and detection $\mathrm{pH} 13$ was difficult to be realized in microchannels without the addition of a strong alkaline solution. However, the condition of reaction $\mathrm{pH} 2$ and detection $\mathrm{pH} 7$ had about three-fourths the fluorescence intensity of the condition of detection $\mathrm{pH} 13$, and was considered to be realized in the microchannel reaction. 


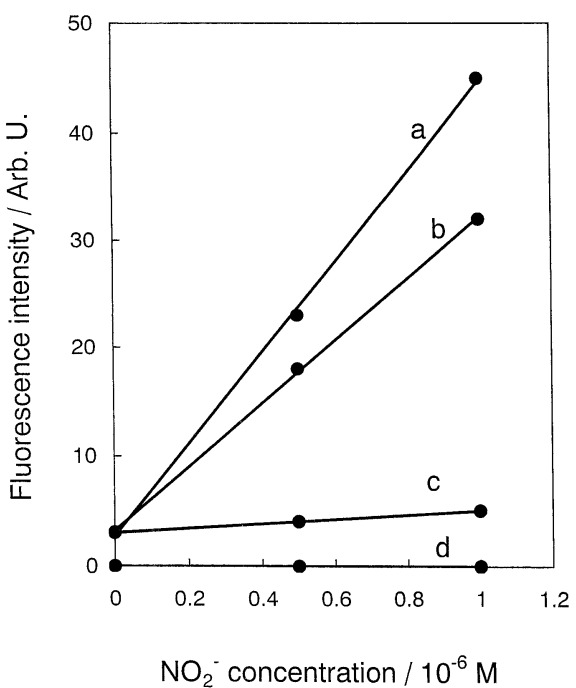

Fig. 2 Fluorescent-intensity dependence on different reaction and detection $\mathrm{pH}$ values in a batch scheme. a, reaction $\mathrm{pH} 2$, detection $\mathrm{pH} 13$; b, reaction $\mathrm{pH} 2$, detection $\mathrm{pH} 7$; c, reaction $\mathrm{pH} 2$, detection $\mathrm{pH} 2$; d, reaction $\mathrm{pH} 6$, detection $\mathrm{pH} 6$.

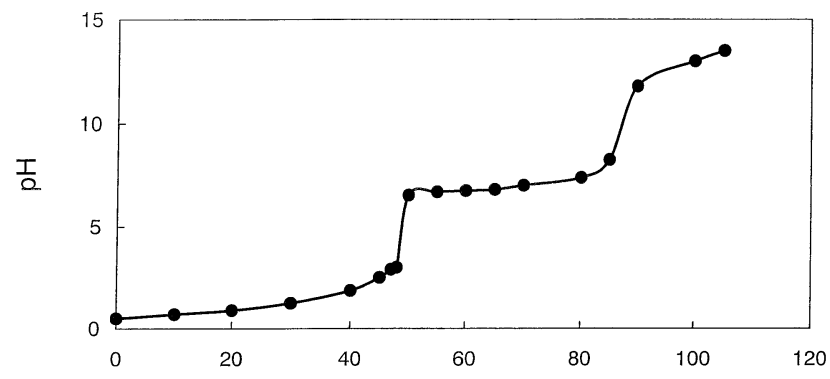

Added $\mathrm{NaOH}$ solution / $\mu \mathrm{L}$

Fig. $3 \mathrm{pH}$ changes of the TEA solution upon the addition of a $5 \mathrm{M}$ $\mathrm{NaOH}$ aqueous solution.

Design of the pH-controlled derivatization and detection of nitrite ions in microchannels

In a gas-absorption experiment, a TEA solution can be used as a gas-absorption solution. TEA is known to be a buffering reagent, and a stable $\mathrm{pH}$ region was expected to be formed by using this buffering effect. The buffering effect of a TEA solution was investigated, and the $\mathrm{pH}$ change of a TEA solution is shown in Fig. 3. The TEA solution was a mixture of $1 \mathrm{ml}$ of 1 $\times 10^{-5} \mathrm{M}$ DAN/0.25 $\mathrm{M} \mathrm{H}_{2} \mathrm{SO}_{4}$ and $1 \mathrm{ml}$ of $3 \%$ (w/v) TEA aqueous solution. The mixture was acidic ( $\mathrm{pH} 0.5)$, and the $\mathrm{pH}$ value changed upon the addition of a $5 \mathrm{M} \mathrm{NaOH}$ aqueous solution. During the addition of 0 to $50 \mu \mathrm{L}$ of a $5 \mathrm{M} \mathrm{NaOH}$ solution, the $\mathrm{pH}$ value gradually changed from 0.5 to 3 , and the first titration point appeared upon the addition of about $50 \mu \mathrm{L}$ of the $\mathrm{NaOH}$ solution. The $\mathrm{pH}$ value then gradually changed from 6.5 to 7.0 during the addition of $80 \mu \mathrm{L}$ of the $\mathrm{NaOH}$ solution. From these results, the $\mathrm{pH}$ change was considered to be small in the $\mathrm{pH}$ range of 6.5 to 7.0. Therefore, because the $\mathrm{pH}$ value was expected to be almost stable at 6.5 to 7.0 , we decided to adjust the $\mathrm{pH}$ value of the mixed sample in the microchannel in this range, where the adequate fluorescence intensity could be obtained.

From the above results concerning a batch scheme, stable and

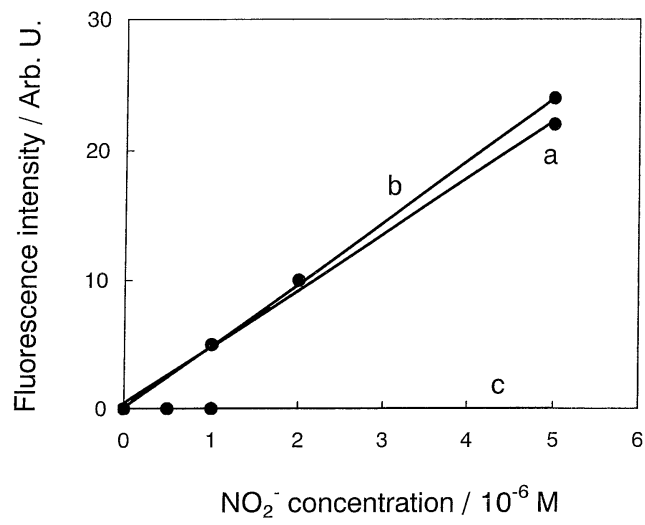

Fig. 4 Comparison of the microchannel reaction and the batch reactions under different $\mathrm{pH}$ conditions. a, microchannel reaction; $\mathrm{b}$, batch reaction (reaction $\mathrm{pH} 2$, detection $\mathrm{pH} 6.8$ ); $\mathrm{c}$, batch reaction (reaction $\mathrm{pH} 6$, detection $\mathrm{pH} 6.8$ ).

efficient derivatization as well as a detection scheme of nitrite ions was designed. In the Y-shaped microchannel used, an equal quantity of the two solutions was considered to be mixed and diluted with each other. We set the final $\mathrm{pH}$ value at 6.8 , where it was expected to be relatively stable. To make the $\mathrm{pH}$ value of a completely mixed solution to be 6.8 , those of the TEA solution and the DAN solution were selected to be 10.5 and 1.0, respectively.

The fluorescence intensity of a mixed solution in the microchannel is shown in Fig. 4. For a comparison, two results of a batch scheme are demonstrated in the same figure. In the microchannel reaction, the sensitivity was almost the same as in the batch reaction, where the reaction $\mathrm{pH}$ was 2 and the detection $\mathrm{pH}$ was made 6.8 upon the addition of an $\mathrm{NaOH}$ aqueous solution. If the two solutions were immediately mixed at the cross point of the Y-shaped microchannel, the $\mathrm{pH}$ value would immediately become 6.8 , and the derivatization reaction would not proceed; thus, a determination plot like line c in Fig. 4 would be obtained. Therefore, in the microchannel reaction, a slightly mixed region, where the $\mathrm{pH}$ value was around 2 , was formed and the derivatization reaction proceeded. The determination limit of nitrite using this microchannel reaction was almost the same as that using the batch reaction, which was in the range of $10^{-7} \mathrm{M}$. However, the reproducibility has so far not been sufficient. This was attributed to the instability of the flow rate in the microchannels. Further developments are necessary for this microchannel reaction before it can be applied to practical uses.

\section{Conclusion}

The effective reaction and detection of nitrite ions with DAN were demonstrated using a $\mathrm{pH}$ gradient generated in a Y-shaped microchannel. This is the first report on a reaction utilizing the $\mathrm{pH}$ gradient formed in a microchannel, and this reaction scheme is only enable in the microchannel, where the specific interface area is much larger than in the usual chemical handlings. By using this sensitive technique for detecting nitrite ions in a TEA solution, which is also used as an $\mathrm{NO}_{2}$ gas-absorption medium, the ultraviolet LED excitation and detection could be integrated on a single microchip. Like IC chips, some microchip devices will be easily incorporated into communications equipment, thus providing both on-site and remote monitoring of 
environmental pollutants, continuous data acquisition and analysis. We have been proposing an on-site monitoring microchip device for air pollutants. ${ }^{25,26}$ Using a porous glass plate as a cover plate of the microchannel chip, $\mathrm{NO}_{2}$ molecules could be absorbed in the absorption solution flowing through the microchannel. Absorbed $\mathrm{NO}_{2}$ molecules exist as nitrite ions, and using the above-mentioned results, a determination method of nitrite ions suitable for microchannels could be constructed. The incorporation of an ultraviolet LED and DAN as a fluorescent excitation source and a fluorescent reagent, respectively, will provide an on-site monitoring microchip device for $\mathrm{NO}_{2}$.

\section{Acknowledgements}

This work was partially supported by NEDO (New Energy and Industrial Technology Development Organization) regional consortium project.

\section{References}

1. A. Manz, N. Graber, and H. M. Widmer, Sens. Actuators, 1990, B1, 244.

2. S. C. Terry, J. H. Jerman, and J. B. Angell, IEEE Trans. Electron Devices, 1979, ED-26, 1880.

3. D. J. Harrison, K. Fluri, K. Seiler, Z. Fan, C. S. Effenhauser, and A. Manz, Science, 1993, 261, 895.

4. C. S. Effenhauser, A. Manz, and H. M. Widmer, Anal. Chem., 1993, 65, 2637.

5. K. Fluri, G. Fitzpatrick, N. Chiem, and D. J. Harrison, Anal. Chem., 1996, 68, 4285.

6. L. D. Hutt, D. P. Glavin, J. L. Bada, and R. A. Mathies, Anal. Chem., 1999, 71, 4000.

7. L. B. Kountny, D. Schmalzing, T. A. Taylor, and M. Fuchs, Anal. Chem., 1996, 68, 18.

8. F. von Heeren, E. Verpoorte, A. Manz, and W. Thormann,
Anal. Chem., 1996, 68, 2044.

9. N. Chiem and D. J. Harrison, Anal. Chem., 1997, 69, 373.

10. C. B. Cohen, E. Chin-Dixon, and T. T. Nikiforov, Anal. Biochem., 1999, 273, 89.

11. J. Cheng, L. C. Waters, and P. Wilding, Anal. Biochem., 1998, 257, 101.

12. W. C. Dunn, S. C. Jacobson, and J. M. Ramsey, Anal. Biochem., 2000, 277, 157.

13. Y.-H. Chen and S.-H. Chen, Electrophoresis, 2000, 21, 165.

14. M. Ueda, H. Nakanishi, and Y. Baba, Materials Science Engineering c: biomimetic, 2000, 12, 33.

15. S. Parinov, V. Barsky, and A. Mirzabekov, Nucleic Acids Res., 1996, 24, 2998.

16. A. A. Stomakhin, V. A. Vasiliskov, and A. D. Mirzabekov, Nucleic Acids Res., 2000, 28, 1193.

17. J. Cheng, M. A. Shoffner, G. E. Hvichia, L. J. Kricka, and P. Wilding, Nucleic Acids Res., 1996, 24, 380.

18. A. T. Woolley, D. Hadley, P. Landre, A. J. Demello, R. A. Mathies, and M. A. Northup, Anal. Chem., 1996, 68, 4081.

19. M. A. Shoffner, J. Cheng, G. E. Hvichia, L. J. Kricka, and P. Wilding, Nucleic Acids Res., 1996, 24, 375.

20. J. Khandurina, T. E. McKnight, S. C. Jacobson, L. C. Waters, R. S. Foote, and J. M. Ramsey, Anal. Chem., 2000, $72,2995$.

21. K. Sato, M. Tokeshi, T. Kitamori, and T. Sawada, Anal. Sci., 1999, 15, 641.

22. M. Tokeshi, T. Minagawa, and T. Kitamori, Anal. Chem., 2000, 72, 1711.

23. M. L. Viriot, B. Mahieuxe, M. C. Carré, and J. C. André, Analusis, 1995, 23, 312.

24. H. Nakanishi, T. Nishimoto, M. Kanai, H. Abe, H. Kuyama, A. Arai, and T. Yoshida, Shimadzu Review, 1999, $56,3$.

25. T. Korenaga, T. Odake, Y. Ono, and L. Rong, Bunseki Kagaku, 2000, 49, 423.

26. T. Sato, H. Susaki, T. Iwata, K. Yamamoto, T. Odake, and T. Korenaga, Trans. IEE of Japan, 2001, in press. 